\begin{tabular}{|l|l|l|l|l|l} 
Revista Praxis & ISSN: $1657-4915$ & Vol. 13 & No. 1 & $69-83$ & enero - junio de 2017 \\
\hline
\end{tabular}

http://dx.doi.org/10.21676/23897856.2069

\title{
EL RENDIMIENTO ACADÉMICO DE ESTUDIANTES UNIVERSITARIOS DEL ÁREA DE PSICOLOGÍA: RELACIONES CON EL CLIMA ORGANIZACIONAL
}

\author{
THE ACADEMIC PERFORMANCE OF UNIVERSITY STUDENTS IN THE AREA OF \\ PSYCHOLOGY: RELATIONS WITH THE ORGANIZATIONAL CLIMATE
}

\author{
Kethy Pérez-Correa ${ }^{\text {iD }}$, Rosario Peley-Bracho² y Elba Saucedo-Ruidíaz ${ }^{3}$
}

\begin{abstract}
RESUMEN
La investigación estuvo orientada a identificar la relación existente entre el rendimiento académico de estudiantes del área de psicología en una universidad pública de Colombia y clima organizacional. Se desarrolló una metodología correlacional y descriptiva con un esquema de campo transaccional y no experimental. El núcleo poblacional estuvo conformado por cuatro (4) directivos, quince (15) docentes y setenta y nueve (79) estudiantes. Para el levantamiento de la información se aplicó como herramienta la encuesta y como instrumentos se diseñaron tres cuestionarios cerrados, con una versión de escala tipo Lickert, conformados por 51 ítems. Los resultados permitieron determinar que las variables estudiadas: clima organizacional y rendimiento académico dentro de la universidad seleccionada para el estudio están estrechamente ligadas, coincidiendo en algunos aspectos entre docentes, estudiantes y directivos y difiriendo en otros. A manera de conclusión, se evidencian tensiones en el clima organizacional como consecuencia de los estilos de dirección, por lo cual, se recomienda la implementación de un conjunto de estrategias para mejorar las condiciones del ambiente laboral y con esto, el rendimiento académico de los estudiantes.
\end{abstract}

Palabras clave: Clima organizacional; rendimiento académico; estudiantes universitarios; área de Psicología.

\section{ABSTRACT}

The research was oriented to identify the relationship between the academic performance of students in the area of psychology in a public university of Colombia and the workplace environment. A correlational and descriptive methodology was developed, with a transactional and non-experimental field scheme. The population nucleus consisted of four (4) managers, fifteen (15) teachers and seventy-nine (79) students. As an instrument, the study used the survey and three closed questionnaires,

Fecha de recepción: marzo 4 de 2017 / Fecha de aceptación: marzo 28 de 2017 / Publicado en línea: abril 19 de 2017 Tipología: Artículo de Investigación Científica y Tecnológica

Para citar este artículo: Pérez, C. K., Peley, B. R. \& Saucedo, R. E. (2017) El rendimiento académico de estudiantes universitarios del área de Psicología: relaciones con el clima organizacional. Praxis, 13(1), 69 - 83. Doi: http://dx.doi.org/10.21676/23897856.2069

\footnotetext{
1. Magíster en Educación con Mención en Gerencia Educativa de la Universidad de Zulia y Magíster en Psicología de la Universidad del Norte. Docente de Tiempo Completo de la Universidad Cooperativa de Colombia - Sede Santa Marta, Colombia. Correo electrónico: kl_perez@hotmail.com ORCID: 0000-0001-8256-7278

2. Doctora en Educación: Perspectivas Históricas, Políticas, Curriculares y de Gestión de la Universidad del Zulia en conjunto con la Universidad de Córdoba. Docente Titular de la Universidad de Zulia. Correo electrónico: rospeley@gmail.com

3. Licenciada en Preescolar de la Universidad del Magdalena. Docente Pedagógica de la Asociación Nacional de Emprendimiento Social y Cultural de Colombia - ASONESHCA. Correo electrónico: ceciliasaucedor@hotmail.com ORCID: 0000-0002-9855-9771
} 
with a Lickert type scale, composed of 51 items. The results allowed to determine that the studied variables: workplace environment and academic performance within the university selected for the study are closely linked, teachers, students and managers coincide in some aspects and differ in others. As a conclusion, there are tensions in the workplace environment as a consequence of the managerial styles. The implementation of a set of strategies aimed at improving the working environment conditions and hence, the academic performance of the students is thus recommended.

Keywords: Workplace environment; academic performance; university students; Psychology area.

\section{INTRODUCCIÓN}

$\mathrm{L}$ a gestión de las organizaciones escolares se convierte en una herramienta efectiva para la interpelación de la calidad educativa (Sánchez, 2010; Sánchez, Linero \& Martínez, 2014). Las áreas de gestión directiva y administrativa introducen elementos para el direccionamiento de los procesos asociados con la cultura institucional, el clima escolar y el talento humano. Son las acciones desarrolladas en estos procesos las que determinan las condiciones del ambiente institucional e influyen en rendimiento de los estudiantes (Keogh, 2008; López, 2013).

El clima organizacional es entendido por Viloria, Pertuz, Daza \& Pedraza (2016) como un conjunto de características de los ambientes laborales, las opiniones de los empleados sobre el funcionamiento de los procesos de la organización y los modelos jerárquicos así como las interacciones entre los elementos organizacionales y personales. Según Cárdenas, Arciniegas \& Barrera (2009) el clima organizacional incide de manera directa o indirecta en los comportamientos de los miembros que componen una organización.

Al respecto Vega, Salazar, Huerta \& Nuñez (2008), sostiene que el clima organizacional es el resultado de las opiniones de los individuos sobre los lineamientos, procesos y políticas en la organización, que se presentan tanto de manera formal como informal, debido a que el hombre se configura como un sujeto complejo (Gómez, 2014).

El concepto de clima organizacional incluye una variedad de significados que ofrecen una visión global a la organización, e íntima una cadena de aspectos multidimensionales propios de la institución. Este compone la personalidad y las características que envuelven las organizaciones y comprende a todos los factores internos que existen en ellas.

En el contexto actual, según Saffle (2006), el clima organizacional es un factor de influencia y peso sobre el desempeño académico en la realidad de la educación, lugar donde se generan las interacciones entre aprendizaje y enseñanza; es por ello que, el brindar un ambiente agradable y motivador al alumno, representa una manera de canalizar que éste haga suyo el conocimiento y de esta forma desarrolle su personalidad.

En este aspecto, según el autor Fernández (2013) en los países latinoamericanos como Chile, Ecuador y Bolivia, se indica que la autoestima y el desarrollo emocional del individuo está entrelazado a su rendimiento académico, y con ello la aparición de consecuencias como la falta de adaptabilidad a las instituciones educativas por parte de los estudiantes. Se presume que las distintas situaciones problemáticas que se presentan en el ambiente de las organizaciones universitarias afectan su clima organizacional aumentando, aún más, la brecha de acoplamiento de los estudiantes con bajo rendimiento.

En relación a lo anterior, se resalta la importancia de un adecuado clima organizacional en las universidades como un elemento básico para la ayuda, adaptación y motivación de los alumnos. Algunas instituciones universitarias se han vuelto una barrera para el estudiante para que supere sus debilidades educativas, desmotivándolos a tal punto que pueda presentarse ausentismo y deserción. 
Jiménez \& López (2009) expresan que “el rendimiento es un constructo multidimensional, determinado por un gran número de variables (inteligencia, motivación, personalidad, etc.) y en el que influyen numerosos factores personales, familiares o escolares, entre otros" (p. 72). En este sentido, es un aspecto que está determinado por algo más que las notas y configurado por factores tanto internos como externos a los estudiantes. Por su parte el desempeño escolar tiene implicaciones en la motivación y autorregulación de los estudiantes (González \& Tourón, 1992), y se deja influenciar por los aspectos imperantes en la institución (Azpiazu, Esnaola \& Ros, 2014).

Analizando la temática en Colombia, según Anzola (2003), se ha evidenciado en las universidades un bajo rendimiento académico en los estudiantes y deserción de las carreras, por diversos problemas en las instituciones educativas universitarias públicas del país como paros, carencia de personal docente, administrativo $\mathrm{u}$ obrero y conflictos internos.

Ante esto se origina la negativa imagen de las universidades para los estudiantes, sin un adecuado clima organizacional que repercute en su rendimiento académico ocasionando frustraciones, apatía, inasistencias, abandono de carrera y/o bajo rendimiento escolar. Es por eso que surge la necesidad de estimular el clima organizacional en las organizaciones educativas universitarias para propiciar la motivación del alumno y, por ende, su desempeño académico.

Ahora bien, para Daza, Charris \& Viloria (2015), las universidades tienen el compromiso de formar un profesional con las competencias necesarias para enfrentarse la realidad social del país; el estudiante debe ser guiado en el proceso académico, mantener un clima organizacional que reluzca en la motivación estudiantil y en su rendimiento. Es así como luego aportará a la ruptura de los sistemas socioeconómicos limitados imperantes en las sociedades actuales (Balza, 2013).

Estos síntomas de la problemática planteada se observan en la institución educativa universitaria, objeto de estudio, donde se destaca que es posible que el clima organizacional y el rendimiento académico de los estudiantes no son los más acordes dado que en muchas instituciones públicas el ambiente laboral es inadecuado, pesado y la motivación es mínima para sus empleados, sumado a las dificultades en la comunicación. Así mismo el bajo rendimiento y la deserción cada vez es mayor en los alumnos, agregando que la organización universitaria manifiesta cierta apatía para motivar al estudiante.

A pesar de que la educación en el país se esfuerza para alcanzar calidad educativa, se puede presumir que de continuar está problemática, se podrían evidenciar estudiantes desmotivados y con bajo rendimiento académico, así como una posible reputación negativa del plantel con un clima organizacional desagradable y pesado para todo los empleados y los estudiantes. (Martínez, 2013) considera que debido a las necesidades de generar aprendizajes significativos se deben promover paradigmas de gestión emergentes que rompan con los modelos pedagógicos tradicionales.

La pretensión del documento es develar las interacciones existentes entre rendimiento académico de estudiantes de Psicología de una universidad de orden público, en la región Caribe de Colombia y el clima laboral.

La importancia de la investigación radica en que permite una comprensión del funcionamiento del clima organizacional en la institución en perspectiva de los resultados académicos de los estudiantes para, desde allí, generar oportunidades y estrategias para el mejoramiento del funcionamiento de los procesos y de la calidad educativa de la institución desde una perspectiva de construcción social, pues comprendiendo la realidad se logra el conocimiento para la transformación de los procesos (Di Marco, 2015). Toda vez que la calidad es un concepto que se construye desde los imaginarios y percepciones de los actores sociales (Viloria, Bertel \& Daza, 2015), y a partir de una cultura innovadora, se generan las estrategias necesarias para la potenciación del componente humano en la organización (Ureña, Jiménez, Mejía, \& Vilarete, 2014). 


\section{METODOLOGÍA}

\section{Tipo de investigación}

La investigación representa dos tipos: descriptiva ya que mide, detalla y describe las características de las variables objeto de estudio (Méndez, 2001) y correlacionar porque (Chávez, 2006) expresa que las investigaciones correlacionales "son aquellas que miden la relación existente entre dos variables".

\section{Diseño de la investigación}

La investigación posee un diseño de campo transversal y no experimental. Según Tamayo \& Tamayo (2006) la investigación de campo “es aquella en que el mismo objeto de estudio sirve como fuente de información para el investigador" (p. 126). Por el contrario, según Hernández, Fernández \& Baptista (2006), en investigaciones no experimentales las variables no son manipuladas de forma deliberada por el investigador; permite explorar la problemática en su ámbito natural para luego analizarlo, más el investigador no posee el control total sobre las variables objeto de estudio.

Por su parte, Chávez (2006) explica que "los diseños transversales o transaccionales poseen como objetivo explorar las influencias del comportamiento de varias o una variable en actores sociales u otros seres vivos, escenarios, sociedades, situaciones, objetos; y desde allí establecer su descripción y características (p.89).

\section{Población y muestra}

La población de la investigación presente es definida por el personal directivo, docente y estudiantil del área de Psicología de la universidad pública analizada. El total de sujetos informantes que conforman la población es de 398 personas, distribuidos de la siguiente forma: 4 directivos, 15 docentes y 379 estudiantes.

Sierra (2005) plantea que una muestra es una representación significativa que reproduce las características de una población, por lo cual se realiza un proceso de tratamiento científico con el objetivo de conseguir hallazgos válidos. Por lo tanto, dado que la población de estudiantes es finita, se tomó una muestra del alumnado utilizando la fórmula para establecer el tamaño de la muestra formulada por Sierra:

$$
\frac{4 x N x P x Q}{E 2(N-1)+4 x P x Q}=n
$$

Donde: $\mathrm{n}=$ tamaño de la muestra; $4=$ constante; $\mathrm{N}$ = tamaño de la población; $\mathrm{P}$ y $\mathrm{Q}=$ probabilidad de éxito o fracaso, con un valor del 50 $\%$ cada uno y E2 = error seleccionado por el investigador, del 10 .

En función a los cálculos realizados para la investigación, se fija la muestra de estudiantes en 79 unidades informantes, a los cuales se les aplica el censo a través de un instrumento diseñado para recopilar los datos desde las percepciones de los sujetos objeto de estudio. Así como a los 4 directivos y 15 docentes.

\section{Técnicas e instrumentos de recolección de datos}

En el presente trabajo resulta importante medir las variables a través de la elaboración de tres (3) cuestionarios de tipo cerrado, utilizando el escalamiento de Likert con las alternativas, siempre, casi siempre, a veces, nunca y casi nunca. El instrumento tiene tres ítems por indicador y un total de 51 preguntas. En cuanto a la presentación del instrumento se organizó de la siguiente forma: 1) identificación de los investigadores, 2) guía para su diligenciamiento, 3) presentación cada campo y sus alternativas para ser respondidos.

\section{Validez y confiabilidad del instrumento}

Una vez diseñado el instrumento, fue sometido a pruebas para verificar su validez y confiabilidad. Se utilizó la validez de contenido que hace referencia a la validación de los instrumentos, sometiéndola a la evaluación de un experto externo desde la opinión de (Hernández et al., 2006); en este sentido, la validez de los instrumentos de la investigación se realizó mediante la evaluación por parte de un total de cinco (5) expertos académicos en el área de educación, 
quienes revisaron la pertinencia de los campos para cada una de las variables, dimensiones e indicadores. Sus observaciones fueron consideradas en la versión final de los instrumentos.

La confiabilidad del instrumento se determinó por el coeficiente Alfa de Cronbach, el cual necesita una aplicación de los instrumentos de recolección y genera valores que oscilan entre uno y cero. Por ente se aplicaron 10 instrumentos en la universidad objeto de estudio, repartidos de la siguiente forma: 2 directores, 3 docentes y 5 estudiantes, logrando una fuerte confiabilidad según el criterio de Cronbach.

\section{Técnicas de análisis de datos}

Para el procesamiento de la información recolectada se elaboró un cuadro de doble entrada. Los datos se muestran en tablas de distribución frecuencial y porcentual para la organización de los indicadores para su tratamiento. Seguidamente, para las discusiones, se utilizaron tablas de distribución de medias aritméticas para cada indicador, variables y dimensiones.

Cabe precisar que los datos se procesaron haciendo uso de la estadística descriptiva, trabajando con el cálculo de frecuencia absoluta, frecuencia relativa, media aritmética; se trabajó solo con los porcentajes, para hallar los resultados por indicadores y por dimensión. Toda vez que las variables objeto de estudio son discretas.

\section{RESULTADOS Y DISCUSIÓN}

En este apartado se plantean los resultados, análisis y discusión de los datos, facilitando así una toma de decisión en la investigación. Se muestra también la explicación del procedimiento estructurado; la organización de la información con la aplicación del instrumento pertinente a los objetivos que persigue el estudio. Así mismo, se procedió a su tabulación con la finalidad de verificar las respuestas y pertinencias de las preguntas formuladas. Los resultados se presentan por variables en las tablas siguientes por cada una de las variables que fueron incluidas en los instrumentos.

Interpretando los resultados en el indicador autoritario explotador, los directores concuerdan en que casi nunca utilizan las amenazas para que el personal de la universidad cumpla sus decisiones, al igual que no suele comunicarse con el personal de la universidad solo para bajar instrucciones; casi nunca confía en el personal de la universidad en relación a los procesos académicos y administrativos. Mientras, los docentes y alumnos difieren de los directores opinando que estos aspectos se suelen cumplir casi siempre en la institución (Tabla 1).

Tabla 1: Tipos de clima organizacional

\begin{tabular}{|l|c|c|c|c|c|c|c|c|c|c|c|c|c|c|c|}
\hline ALTERNATIVA & \multicolumn{3}{|c|}{ SIEMPRE } & \multicolumn{3}{|c|}{ CASI SIEMPRE } & \multicolumn{3}{|c|}{ A VECES } & \multicolumn{3}{c|}{ CASI NUNCA } & \multicolumn{3}{|c|}{ NUNCA } \\
\hline \multicolumn{1}{|c|}{ POBLACIÓN } & Dir & Doc & Est & Dir & Doc & Est & Dir & Doc & Est & Dir & Doc & Est & Dir & Doc & Est \\
\hline INDICADORES & $\mathbf{\%}$ & $\mathbf{\%}$ & $\mathbf{\%}$ & $\mathbf{\%}$ & $\mathbf{\%}$ & $\mathbf{\%}$ & $\mathbf{\%}$ & $\mathbf{\%}$ & $\mathbf{\%}$ & $\mathbf{\%}$ & $\mathbf{\%}$ & $\mathbf{\%}$ & $\mathbf{\%}$ & $\mathbf{\%}$ & $\mathbf{\%}$ \\
\hline $\begin{array}{l}\text { Autoritario } \\
\text { explorador }\end{array}$ & 0 & 26 & 3 & 0 & 27 & 41 & 25 & 20 & 25 & 75 & 17 & 17 & 0 & 10 & 11 \\
\hline $\begin{array}{l}\text { Autoritario } \\
\text { paternalista }\end{array}$ & 0 & 17 & 10 & 0 & 18 & 13 & 50 & 29 & 25 & 50 & 23 & 23 & 0 & 13 & 19 \\
\hline $\begin{array}{l}\text { Participativo } \\
\text { consultivo }\end{array}$ & 25 & 13 & 8 & 50 & 11 & 10 & 25 & 17 & 17 & 0 & 39 & 48 & 0 & 20 & 17 \\
\hline $\begin{array}{l}\text { Participativo en } \\
\text { grupo }\end{array}$ & 0 & 5 & 4 & 50 & 9 & 8 & 25 & 13 & 13 & 25 & 39 & 53 & 0 & 33 & 23 \\
\hline $\begin{array}{l}\text { RESUMEN } \\
\text { GENERAL }\end{array}$ & $\mathbf{6}$ & $\mathbf{1 5}$ & $\mathbf{6}$ & $\mathbf{2 5}$ & $\mathbf{1 6}$ & $\mathbf{1 8}$ & $\mathbf{3 1}$ & $\mathbf{2 0}$ & $\mathbf{2 0}$ & $\mathbf{3 8}$ & $\mathbf{3 0}$ & $\mathbf{3 5}$ & $\mathbf{0}$ & $\mathbf{1 9}$ & $\mathbf{1 8}$ \\
\hline
\end{tabular}

Fuente: elaboración propia 
En el indicador autoritario paternalista, para los directores, en igualdad de opiniones que a veces y casi nunca la toma de decisiones se concentra en la gerencia, al igual que, no suele dar sanciones como medio de presión para alcanzar sus fines, tampoco suele tratar al personal de la universidad con condescendencia. Mientras, que los docentes y alumnos opinan que estos aspectos se suelen cumplir a veces en la institución.

En el indicador participativo consultivo, para los directores, casi siempre los objetivos institucionales son elegidos por todo el personal que labora en la universidad ofrece recompensas al personal docente por un trabajo bien realizado como medio de motivación laboral y piensa que se respira un ambiente de trabajo dinámico y proactivo en la universidad. Mientras que los docentes y alumnos opinan que estos aspectos casi nunca se suelen cumplir en la institución.

Desde el indicador participativo en grupo se señala, desde los directores, que casi siempre el trabajo en equipo es el método utilizado en la universidad para buscar soluciones a los conflictos presentados, al igual de que en la universidad se propicia el trabajo en equipo para el logro de los objetivos institucionales. La comunicación entre los miembros de la institución es asertiva. Mientras, docentes y estudiantes opinan que estos aspectos casi nunca se suelen cumplir en la institución. A modo de cierre para la dimensión tipos en la universidad objeto de estudio, el tipo de clima predominante es el autoritario paternalista con la sumatoria de las tres poblaciones y el menos usado es el participativo en grupo (Tabla 1).

Ante estos hechos es oportuno señalar el planteamiento de Pérez (2006) en el que sostiene que el clima organizacional muestra hoy en día gran relevancia dentro de las organizaciones educativas; es por ello que es de suma importancia el estudio de los tipos de clima organizacional que se desarrollan en una institución, para determinar si esta es la más adecuada para el contexto y ambiente de la entidad. Para el autor Likert (citado por Robbins 2004), los tipos de clima organizacional generados por los directivos o gerentes, está envuelto en dos sistemas: el sistema I (negativo) de cortes autoritarios paternalistas y autoritarios explotadores, y el sistema II (positivo) participativos en grupos y participativos consultivos.

Acotando a lo anterior, se encuentra que dependiendo de las diferentes circunstancias en una organización se presentan diversas dimensiones del clima organizacional. En este sentido estos elementos son lo que configuran un macroclima en el ambiente laboral (Robbins, 2004).

Como se evidencia en la distribución estadística, se plantean los resultados de la tabla estadística $\mathrm{N}^{\circ} 2$, de la dimensión, en su indicador estructura, se destaca que los directores consideran que en proporciones iguales siempre y casi siempre dan a conocer la misión, visión y reglas de la organización. De la misma manera, relaciona el trabajo que desarrolla con los objetivos de la organización, y se siente satisfecho y comprometido con las directrices estratégicas de la organización. Mientras, para que docentes y estudiantes opinan que estos aspectos se suelen cumplir solo algunas veces en la institución.

Ahora bien, observando los resultados del indicador recompensa, interpretando a los directores, a veces reciben una compensación salarial acorde con sus habilidades y experiencia; solo en ocasiones está satisfecho con los beneficios que ofrece la organización, pues solo a veces se distribuye entre los miembros de la organización los premios y reconocimientos en forma justa. Por el contrario los docentes opinan que estos aspectos se suelen cumplir casi siempre en la institución, y según los estudiantes estos aspectos a veces se cumplen. 
Tabla 2: Dimensiones del clima organizacional

\begin{tabular}{|l|c|c|c|c|c|c|c|c|c|c|c|c|c|c|c|}
\hline \multirow{2}{*}{ ALTERNATIVA } & \multicolumn{3}{|c|}{ SIEMPRE } & \multicolumn{3}{c|}{ CASI SIEMPRE } & \multicolumn{3}{c|}{ A VECES } & \multicolumn{3}{c|}{ CASI NUNCA } & \multicolumn{3}{c|}{ NUNCA } \\
\hline \multicolumn{1}{|c|}{ POBLACIÓN } & Dir & Doc & Est & Dir & Doc & Est & Dir & Doc & Est & Dir & Doc & Est & Dir & Doc & Est \\
\hline INDICADORES & $\%$ & $\%$ & $\%$ & $\%$ & $\%$ & $\%$ & $\%$ & $\%$ & $\%$ & $\%$ & $\%$ & $\%$ & $\%$ & $\%$ & $\%$ \\
\hline Estructura & 50 & 15 & 12 & 50 & 20 & 18 & 0 & 30 & 59 & 0 & 23 & 22 & 0 & 13 & 7 \\
\hline Recompensa & 0 & 28 & 10 & 25 & 42 & 22 & 50 & 13 & 59 & 25 & 13 & 18 & 0 & 7 & 9 \\
\hline Relaciones & 0 & 0 & 6 & 25 & 3 & 2 & 50 & 13 & 20 & 25 & 81 & 52 & 0 & 3 & 21 \\
\hline Cooperación & 25 & 10 & 11 & 50 & 9 & 25 & 25 & 40 & 41 & 0 & 30 & 3 & 0 & 11 & 17 \\
\hline Conflicto & 25 & 4 & 10 & 25 & 8 & 19 & 50 & 23 & 25 & 0 & 53 & 22 & 0 & 13 & 24 \\
\hline $\begin{array}{l}\text { RESUMEN } \\
\text { GENERAL }\end{array}$ & $\mathbf{2 0}$ & $\mathbf{1 1}$ & $\mathbf{1 0}$ & $\mathbf{3 5}$ & $\mathbf{1 6}$ & $\mathbf{1 7}$ & $\mathbf{3 5}$ & $\mathbf{2 4}$ & $\mathbf{4 1}$ & $\mathbf{1 0}$ & $\mathbf{4 0}$ & $\mathbf{2 3}$ & $\mathbf{0}$ & $\mathbf{9}$ & $\mathbf{1 6}$ \\
\hline
\end{tabular}

Fuente: elaboración propia.

De esta forma, en el indicador relaciones, se observa que los directores piensan que a veces pueden contar con los miembros de la organización cuando los necesiten, al igual que solo en algunas ocasiones confían en los miembros de la organización; a veces guarda una relación de apoyo entre los miembros de la organización, en cuanto a las actividades académicas y extracurriculares. Mientras que los docentes y estudiantes opinan que estos aspectos casi nunca se cumplen en la institución.

En el indicador cooperación los directores coinciden en que casi siempre reciben cooperación de los miembros de la institución cuando lo requieren, al igual que les exigen un rendimiento alto a los miembros de la organización; apoyan a sus compañeros de trabajo cuando estos lo ameritan. Mientras, los docentes y estudiantes opinan que estos aspectos se suelen cumplir solo a veces en la institución.

En este sentido, el indicador conflicto, los directores señalan que a veces se presentan conflictos en la universidad entre los gerentes y el personal, al igual que en ocasiones, en la organización, existen conflictos entre el personal docente. Solo a veces sabe resolver rápidamente los conflictos internos de la organización. Los docentes opinan que estos aspectos casi nunca se suelen cumplir en la institución, y según los estudiantes estos aspectos a veces se cumplen. A modo de cierre, para la condición dimensiones, el indicador por tendencia más positiva es el de recompensa, mientras que los que se mantienen en una inclinación negativa se encuentran las relaciones y el conflicto.

Ante estos hechos es oportuno señalar el planteamiento de García (2009) en el que expresa que en el clima organizacional las dimensiones se definen como los elementos o caracterizaciones que tienen alguna incidencia en la conducta de las personas y que son susceptibles de ser evaluadas. En este sentido, el clima organizacional es una configuración compleja donde confluyen diversos elementos y variables, como el entorno social de las organizaciones.

Se encuentran los autores clásicos Litwin \& Stinger (1978) quienes en su teoría reflexionan sobre cómo el entorno y la estructura de la organización inciden en el clima organizacional, indicando a su vez que este incide en la conducta de los individuos.

Se presenta la tabla estadística $\mathrm{N}^{\circ} 3$, de la dimensión aspectos que intervienen en el rendimiento académico, en su indicador insumos escolares, se puede interpretar que los directores a veces evidencian que los alumnos cuentan con los útiles y textos escolares correspondientes a la carrera, al igual que sólo algunas veces la biblioteca de la universidad cuenta con una amplia gama de 
libros. A veces la universidad proporciona al estudiante aulas virtuales para que estos puedan realizar sus actividades académicas. Mientras los docentes opinan que estos aspectos se suelen cumplir, casi nunca en la institución y según los estudiantes estos aspectos a veces se cumplen.

Tabla 3: Aspectos que intervienen en el rendimiento académico

\begin{tabular}{|l|c|c|c|c|c|c|c|c|c|c|c|c|c|c|c|}
\hline ALTERNATIVA & \multicolumn{3}{|c|}{ SIEMPRE } & \multicolumn{1}{|c|}{ CASI SIEMPRE } & \multicolumn{3}{|c|}{ A VECES } & \multicolumn{3}{|c|}{ CASI NUNCA } & \multicolumn{3}{c|}{ NUNCA } \\
\hline \multicolumn{1}{|c|}{ POBLACIÓN } & Dir & Doc & Est & Dir & Doc & Est & Dir & Doc & Est & Dir & Doc & Est & Dir & Doc & Est \\
\hline INDICADORES & $\%$ & $\mathbf{\%}$ & $\mathbf{\%}$ & $\mathbf{\%}$ & $\mathbf{\%}$ & $\mathbf{\%}$ & $\mathbf{\%}$ & $\mathbf{\%}$ & $\mathbf{\%}$ & $\mathbf{\%}$ & $\mathbf{\%}$ & $\mathbf{\%}$ & $\mathbf{\%}$ & $\mathbf{\%}$ & $\mathbf{\%}$ \\
\hline $\begin{array}{l}\text { Insumos } \\
\text { escolares }\end{array}$ & 0 & 13 & 7 & 25 & 17 & 28 & 75 & 18 & 32 & 0 & 29 & 20 & 0 & 23 & 12 \\
\hline $\begin{array}{l}\text { Motivación } \\
\text { escolar }\end{array}$ & 0 & 10 & 7 & 25 & 20 & 13 & 25 & 26 & 28 & 50 & 27 & 42 & 0 & 17 & 13 \\
\hline Autocontrol & 0 & 13 & 5 & 0 & 20 & 10 & 25 & 39 & 56 & 50 & 11 & 15 & 25 & 17 & 12 \\
\hline $\begin{array}{l}\text { Habilidades } \\
\text { sociales }\end{array}$ & 25 & 2 & 13 & 25 & 9 & 17 & 50 & 16 & 23 & 0 & 44 & 29 & 0 & 16 & 18 \\
\hline $\begin{array}{l}\text { RESUMEN } \\
\text { GENERAL }\end{array}$ & $\mathbf{6}$ & $\mathbf{1 0}$ & $\mathbf{8}$ & $\mathbf{1 9}$ & $\mathbf{6 6}$ & $\mathbf{1 7}$ & $\mathbf{4 4}$ & $\mathbf{2 5}$ & $\mathbf{3 5}$ & $\mathbf{2 5}$ & $\mathbf{2 8}$ & $\mathbf{2 6}$ & $\mathbf{6}$ & $\mathbf{1 8}$ & $\mathbf{1 4}$ \\
\hline
\end{tabular}

Fuente: elaboración propia

En los resultados del indicador motivación escolar se observa para los directores que casi nunca se propician en la universidad actividades especiales para motivar al alumno, al igual que no suelen percibir que el estudiante se sienta apoyado y acompañado por sus docentes en toda su carrera; casi nunca los docentes motivan al estudiante a través de asesorías personales. Mientras que los docentes y estudiantes opinan que estos aspectos se suelen cumplir sólo a veces en la institución.

En el indicador autocontrol se destacan las opiniones de los directores, los cuales plantean que casi nunca se utiliza la inteligencia emocional en la organización para resolver conflictos entre alumnos y docentes; al igual que los alumnos no suelen sentir control y confianza del entorno universitario que le rodea, casi nunca los estudiantes poseen la capacidad de modular y controlar sus acciones en el plantel de una forma apropiada para su edad. Mientras que los docentes y estudiantes opinan que estos aspectos se suelen cumplir solo a veces en la institución.

En el indicador habilidades sociales se observa que los directores consideran que a veces los docentes son aislados y poco receptivos con los estudiantes, al igual que solo en algunas ocasiones los alumnos evidencian problemas para relacionarse en el entorno universitario, a veces los estudiantes y el personal de la institución se relacionan de forma adecuada. Mientras, docentes y estudiantes opinan que estos aspectos se suelen cumplir casi nunca en la institución. Presentando como cierre de la dimensión en los aspectos que intervienen en el rendimiento académico en la universidad objeto de estudio todos mantienen tendencia negativa, sin embargo las habilidades sociales poseen mayor debilidad.

Es oportuno señalar el planteamiento de Mizola (2005) quien expresa que cuando existe la necesidad de medir el desempeño académico y definir las estrategias para su mejoramiento deben revisarse los elementos que pueden tener alguna influencia en el mismo, considerando que el rendimiento académico es una variable que depende de diversos factores.

A partir de lo anterior, se encuentra Edel (2003) quien señala cuatro aspectos que intervienen en el rendimiento académico: los insumos, la motivación escolar, el autocontrol del alumno 
y las habilidades sociales. En este sentido estos aspectos requieren ser valorados en los contextos educativos para determinar sus incidencias en el desempeño académico.

En este momento, se plantean los resultados de la tabla estadística $\mathrm{N}^{\circ} 4$, de la dimensión factores que influyen en el rendimiento académico, en su indicador fisiológicos, se puede interpretar que los directores consideran que a veces la universidad brinda al estudiante apoyo en servicios médicos, al igual que solo en algunas ocasiones la universidad aporta alimentación diaria a los alumnos a través del comedor estudiantil. A veces se toma en cuenta en la organización las necesidades físicas especiales de los alumnos. Mientras que los docentes opinan que estos aspectos se suelen cumplir casi nunca en la institución, y según los estudiantes estos aspectos a veces se cumplen.

Tabla 4: Factores que influyen en el rendimiento académico

\begin{tabular}{|l|c|c|c|c|c|c|c|c|c|c|c|c|c|c|c|}
\hline \multirow{2}{*}{ ALTERNATIVA } & \multicolumn{3}{|c|}{ SIEMPRE } & \multicolumn{3}{|c|}{ CASI } & \multicolumn{3}{c|}{ A VECES } & \multicolumn{3}{c|}{ CASI NUNCA } & \multicolumn{3}{c|}{ NUNCA } \\
\hline \multicolumn{1}{|c|}{ POBLACIÓN } & Dir & Doc & Est & Dir & Doc & Est & Dir & Doc & Est & Dir & Doc & Est & Dir & Doc & Est \\
\hline INDICADORES & $\%$ & $\%$ & $\%$ & $\mathbf{\%}$ & $\mathbf{\%}$ & $\mathbf{\%}$ & $\mathbf{\%}$ & $\%$ & $\%$ & $\%$ & $\%$ & $\%$ & $\%$ & $\%$ & $\%$ \\
\hline Fisiológicos & 0 & 8 & 15 & 25 & 20 & 23 & 50 & 32 & 30 & 25 & 27 & 20 & 0 & 12 & 13 \\
\hline Pedagógicos & 25 & 17 & 13 & 50 & 48 & 23 & 25 & 17 & 53 & 0 & 10 & 8 & 0 & 8 & 4 \\
\hline Sociológicos & 0 & 12 & 10 & 50 & 15 & 9 & 25 & 56 & 40 & 25 & 5 & 30 & 0 & 10 & 11 \\
\hline Desmotivación & 25 & 27 & 12 & 50 & 52 & 82 & 25 & 22 & 6 & 0 & 0 & 0 & 0 & 0 & 0 \\
\hline $\begin{array}{l}\text { RESUMEN } \\
\text { GENERAL }\end{array}$ & $\mathbf{1 3}$ & $\mathbf{6 4}$ & $\mathbf{1 3}$ & $\mathbf{4 4}$ & $\mathbf{3 4}$ & $\mathbf{3 4}$ & $\mathbf{3 1}$ & $\mathbf{3 2}$ & $\mathbf{3 1}$ & $\mathbf{1 3}$ & $\mathbf{1 1}$ & $\mathbf{1 5}$ & $\mathbf{0}$ & $\mathbf{7}$ & $\mathbf{7}$ \\
\hline
\end{tabular}

Fuente: elaboración propia

Analizando los resultados del indicador pedagógicos se observa en los directores que casi siempre se promueve en la institución el uso de las TICs dentro de la universidad, al igual que los materiales didácticos utilizados en las clases son de primera calidad. Se fomenta en los alumnos de la organización el reconocimiento a los más altos índices académicos. Los docentes coinciden con los directivos y opinan que estos aspectos se suelen cumplir casi siempre en la institución, y según los estudiantes estos aspectos a veces se cumplen.

De esta forma, en el indicador sociológicos, se plantea que los directores coinciden en que casi siempre se realizan actividades en la universidad que involucren a las familias de los alumnos para insertarlos en el proceso educativo de sus hijos, al igual que se crean actividades sociales entre los alumnos para mantener una buena relación entre ellos. La universidad facilita y estimula la convivencia entre docentes y alumnos. Mientras que los docentes y alumnos opinan que estos aspectos se suelen cumplir solo a veces en la institución.

Continuando con el indicador desmotivación, se observa que los directores coinciden en que casi siempre la estructura administrativa y burocrática de la universidad frena e incomoda al estudiante, al igual que el ambiente universitario donde se desenvuelve el estudiante es tenso y pesado; aunado a esto, los conflictos entre el personal de la universidad desmotivan a los estudiantes. Sin embargo, los docentes y estudiantes coinciden con los directores al opinar que estos aspectos se suelen cumplir solo algunas veces en la institución. Para modo de cierre dentro de la universidad objeto de estudio, de los factores que influyen en el rendimiento académico, el que presenta mayores fortalezas es el pedagógico, mostrando debilidad en todos los demás, principalmente en la desmotivación. 
Es necesario mencionar el planteamiento de Fernández (2013), quien señala que el éxito académico y la satisfacción de los estudiantes con la enseñanza universitaria proviene de la satisfacción del estudiante; lo cual varía directamente con el éxito que obtienen en aquellas asignaturas propias de la carrera. Sin embargo, existen factores que pueden entorpecer la satisfacción del estudiante, generando un rendimiento académico por debajo de lo deseado como lo son: fisiológicos, pedagógicos, sociológicos y desmotivación.

Mizola (2005), al señalar la determinación de los factores que afectan al rendimiento académico en los estudiantes de educación superior, llega a la conclusión de que uno de los factores es el esfuerzo efectivo desarrollado por el educando, así como sus competencias y saberes anteriores; $\sin$ embargo, también existen factores determinantes como el ambiente del alumno y aspectos internos de la universidad donde estudia. A partir de las exploraciones investigativas de estos factores se gestiona el conocimiento en las organizaciones (Agudelo, Martínez \& Ortiz, 2013).

Según la presente distribución estadística de la tabla 5 como resultados de la variable clima organizacional en su dimensión tipos, se observa en la opción siempre un $9 \%$, para la alternativa casi siempre un $20 \%$, en la opción a veces 24 $\%$, para casi nunca $35 \%$, mientras que nunca un $12 \%$ de las respuestas.

En este mismo orden de ideas, como resultados de la variable clima organizacional en su dimensión dimensiones, se presenta la segunda dimensión con la alternativa siempre un $14 \%$, para casi siempre un $23 \%$, en a veces $33 \%$, luego casi nunca un $24 \%$ y por último la opción nunca con un $8 \%$ de las respuestas.

Tabla 5: Clima organizacional

\begin{tabular}{|c|c|c|c|c|c|c|c|c|c|c|c|c|c|c|c|}
\hline ALTERNATIVA & \multicolumn{3}{|c|}{ SIEMPRE } & \multicolumn{3}{|c|}{$\begin{array}{c}\text { CASI } \\
\text { SIEMPRE }\end{array}$} & \multicolumn{3}{|c|}{ A VECES } & \multicolumn{3}{|c|}{ CASI NUNCA } & \multicolumn{3}{|c|}{ NUNCA } \\
\hline POBLACIÓN & Dir & Doc & Est & Dir & Doc & Est & Dir & Doc & Est & Dir & Doc & Est & Dir & Doc & Est \\
\hline INDICADORES & $\%$ & $\%$ & $\%$ & $\%$ & $\%$ & $\%$ & $\%$ & $\%$ & $\%$ & $\%$ & $\%$ & $\%$ & $\%$ & $\%$ & $\%$ \\
\hline Tipos & 6 & 15 & 6 & 25 & 16 & 18 & 31 & 20 & 20 & 38 & 30 & 35 & 0 & 19 & 18 \\
\hline $\begin{array}{l}\text { Resumen de la } \\
\text { dimensión }\end{array}$ & \multicolumn{3}{|c|}{9} & \multicolumn{3}{|c|}{20} & \multicolumn{3}{|c|}{24} & \multicolumn{3}{|c|}{35} & \multicolumn{3}{|c|}{12} \\
\hline Dimensiones & 20 & 11 & 10 & 35 & 16 & 17 & 35 & 24 & 41 & 10 & 40 & 23 & 0 & 9 & 16 \\
\hline $\begin{array}{l}\text { RESUMEN DE LA } \\
\text { DIMENSIÓN }\end{array}$ & \multicolumn{3}{|c|}{14} & \multicolumn{3}{|c|}{23} & \multicolumn{3}{|c|}{33} & \multicolumn{3}{|c|}{24} & \multicolumn{3}{|c|}{8} \\
\hline $\begin{array}{l}\text { RESUMEN } \\
\text { GENERAL }\end{array}$ & 26 & 26 & 16 & 60 & 32 & 35 & 66 & 44 & 61 & 48 & 70 & 58 & 9 & 28 & 34 \\
\hline
\end{tabular}

Fuente: elaboración propia

En la distribución estadística de la tabla 6, como resultados de la variable rendimiento académico, en su dimensión aspectos que inciden en el rendimiento académico, se observa en el resumen de la dimensión en la opción siempre un $8 \%$, para casi siempre un $29 \%$, en a veces $31 \%$, casi nunca $20 \%$, y nunca con un $12 \%$ de las respuestas.

Y como resultados de la variable rendimiento académico, en su dimensión factores que influyen en el rendimiento académico, la alternativa 
siempre tiene un $26 \%$, casi siempre un $31 \%$, a veces $29 \%$, casi nunca un $11 \%$ y, por último, la opción nunca un $3 \%$ de las respuestas.

En este sentido, se utiliza la correlación de Spearman para medir en el grado de relación entre clima organizacional y rendimiento académico. Luego de concluir el análisis de los resultados y el proceso estadístico, se procedió a establecer la correlación existente entre las variables del estudio y, de esta forma, poder medir el grado de asociación de las dos variables. La fuerza de una relación entre dos se puede medir con el coeficiente "Rho"; es decir mediante el Coeficiente Spearman cuyo valor va desde -1 para la correlación negativa perfecta y hasta +1 para la correlación positiva perfecta, tal como se refleja la tabla 7 .

Tabla 6: Rendimiento académico

\begin{tabular}{|c|c|c|c|c|c|c|c|c|c|c|c|c|c|c|c|}
\hline ALTERNATIVA & \multicolumn{3}{|c|}{ SIEMPRE } & \multicolumn{3}{|c|}{$\begin{array}{c}\text { CASI } \\
\text { SIEMPRE }\end{array}$} & \multicolumn{3}{|c|}{ A VECES } & \multicolumn{3}{|c|}{ CASI NUNCA } & \multicolumn{3}{|c|}{ NUNCA } \\
\hline POBLACIÓN & Dir & Doc & Est & Dir & Doc & Est & Dir & Doc & Est & Dir & Doc & Est & Dir & Doc & Est \\
\hline DIMENSIÓN & $\%$ & $\%$ & $\%$ & $\%$ & $\%$ & $\%$ & $\%$ & $\%$ & $\%$ & $\%$ & $\%$ & $\%$ & $\%$ & $\%$ & $\%$ \\
\hline $\begin{array}{l}\text { Aspectos que } \\
\text { intervienen en } \\
\text { el rendimiento } \\
\text { académico }\end{array}$ & 6 & 10 & 8 & 19 & 66 & 17 & 44 & 25 & 35 & 25 & 28 & 26 & 6 & 18 & 14 \\
\hline $\begin{array}{l}\text { RESUMEN DE LA } \\
\text { DIMENSIÓN }\end{array}$ & \multicolumn{3}{|c|}{8} & \multicolumn{3}{|c|}{29} & \multicolumn{3}{|c|}{31} & \multicolumn{3}{|c|}{20} & \multicolumn{3}{|c|}{12} \\
\hline $\begin{array}{l}\text { Factores que } \\
\text { influyen en el } \\
\text { rendimiento } \\
\text { académico }\end{array}$ & 13 & 64 & 13 & 44 & 34 & 34 & 31 & 32 & 32 & 13 & 11 & 15 & 0 & 7 & 7 \\
\hline $\begin{array}{l}\text { RESUMEN DE LA } \\
\text { DIMENSIÓN }\end{array}$ & \multicolumn{3}{|c|}{26} & \multicolumn{3}{|c|}{31} & \multicolumn{3}{|c|}{29} & \multicolumn{3}{|c|}{11} & \multicolumn{3}{|c|}{3} \\
\hline $\begin{array}{l}\text { RESUMEN } \\
\text { GENERAL }\end{array}$ & 19 & 74 & 21 & 63 & 97 & 51 & 75 & 57 & 67 & 38 & 39 & 41 & 6 & 25 & 21 \\
\hline
\end{tabular}

Fuente: elaboración propia

Tabla 7: Correlación de las variables

\begin{tabular}{|c|l|c|c|}
\hline \multirow{2}{*}{ Rh de Spearman } & Clima organizacional & Rendimiento académico \\
\cline { 2 - 4 } & $\begin{array}{l}\text { Clima organizacional } \\
\text { Coeficiente de } \\
\text { correlación }\end{array}$ & 1,000 &, $711^{*}$ \\
\hline \multirow{2}{*}{} & $\begin{array}{l}\text { Rendimiento } \\
\text { académico } \\
\text { Coeficiente de } \\
\text { correlación }\end{array}$ & 101 &, 000 \\
&, 000 & 101 \\
\hline
\end{tabular}

Fuente: elaboración propia 
Luego de aplicar el Coeficiente de Spearman se obtuvo el valor de 0,711 significativamente positivo bilateral, lo que demuestra que cuando se elevan los valores de la variable clima organizacional aumenta de manera positiva y significante la variable rendimiento académico; el valor obtenido para la significancia bilateral es menor que la probabilidad de error esperada 0,01 , cuya relación de valores de la otra variable clima organizacional es significativa -estadísticamente- $\mathrm{y}$ viceversa. Lo que demuestra la importancia de un adecuado clima organización dentro de la institución objeto de estudio para mejorar el rendimiento académico de los estudiantes.

\section{CONCLUSIONES}

Al finalizar la presentación de los resultados que conforman el sumario de investigación referido al clima organizacional y el rendimiento académico de los estudiantes, y después de haber ejecutado el procesamiento estadístico de los datos recolectados mediante los instrumentos aplicados, se pueden presentar las siguientes anotaciones:

En función de la tipología de clima organizacional presente en la Universidad se evidencia que el director utiliza las amenazas para que el personal de la universidad cumpla sus decisiones, al igual que suele comunicarse con el personal de la universidad sólo para bajar instrucciones. Se evidencia que poco confía en el personal de la universidad en relación a los procesos académicos y administrativos. Tampoco suele tratar al personal de la universidad con condescendencia.

Adicionalmente no se respira un ambiente de trabajo dinámico y proactivo en la universidad, al igual que no suele ofrecer recompensas al personal docente por un trabajo bien realizado. Entre otras cosas, el trabajo en equipo no se configura como el método utilizado en la universidad para buscar soluciones a los conflictos presentados y, por lo tanto, no se propicia el trabajo en equipo. La comunicación entre los miembros de la institución es poco asertiva. Para la dimensión tipos, en la universidad objeto de estudio, el tipo de clima organizacional predominante es el autoritario paternalista con la sumatoria de las tres poblaciones. El menos usado es el participativo en grupo.

En términos de las dimensiones del clima organizacional en el área de Psicología se concluye que en pocas ocasiones el personal de la universidad recibe una compensación salarial acorde con sus habilidades y experiencia, al igual que solo en ocasiones están satisfechos con los beneficios que ofrece la organización. Solo en algunas ocasiones se distribuye entre los miembros de la organización los premios y reconocimientos en forma justa.

Las poblaciones estudiadas muy poco pueden contar con el apoyo de los miembros de la organización cuando los necesite, al igual que no suelen confiar en los miembros de la organización. El nivel de exigencia en términos de rendimiento es alto para los miembros de la organización. Cabe señalar que se presentan conflictos en la universidad entre los gerentes y el personal; en ocasiones en la organización existen conflictos entre el personal docente. Es así que para la dimensión dimensiones el indicador por tendencia más positiva es el de recompensa, mientras que los que se mantienen en una inclinación negativa se encuentran relaciones y conflicto.

Desde la perspectiva de los aspectos que intervienen en el rendimiento académico de los estudiantes del área de psicología solo en ocasiones los alumnos cuentan con los útiles y textos escolares correspondientes a la carrera, y la biblioteca de la universidad no cuenta con una amplia gama de libros. En este sentido, solo a veces la universidad proporciona al estudiante aulas virtuales para que estos puedan realizar sus actividades académicas. También se destaca que no se propician en la universidad actividades especiales para motivar al alumno, y por lo mismo el estudiante no se siente apoyado y acompañado por sus docentes en toda su carrera.

A esto se le suma que la inteligencia emocional en la organización no se usa como una herramienta efectiva para resolver conflictos entre alumnos y docentes. Los alumnos a veces sienten control y confianza del entorno universitario 
que los rodea, profundizando en esta dinámica; en pocas ocasiones los estudiantes poseen la capacidad de modular y controlar sus acciones en el plantel de una forma apropiada para su edad. Los docentes se muestran aislados y poco receptivos con los estudiantes, a su vez que los alumnos evidencian problemas para relacionarse en el entorno universitario. Los estudiantes y el personal de la institución muy poco se relacionan de forma adecuada. Como cierre de la dimensión en la universidad objeto de estudio todos mantienen tendencia negativa, sin embargo, las habilidades sociales poseen mayor debilidad.

Por otro lado, los factores que influyen en el rendimiento académico de los estudiantes destacan que la universidad en momentos brinda al estudiante apoyo en servicios médicos, al igual que, en algunas ocasiones, aporta alimentación diaria a los alumnos a través del comedor estudiantil. También, en ocasiones, se realizan actividades en la universidad que involucren a las familias de los alumnos para insertarlos en el proceso educativo de sus hijos, y crean en algunos escenarios actividades sociales entre los alumnos para mantener una buena relación entre ellos.

La estructura administrativa y burocrática de la universidad se caracteriza por frenar e incomodar al estudiante. Así mismo el ambiente universitario donde se desenvuelve el estudiante suele ser tenso y pesado, y por lo tanto los conflictos entre el personal de la universidad desmotivan a los estudiantes. A modo de cierre, dentro de la universidad objeto de estudio, los factores que influyen en el rendimiento académico; el factor con más fortalezas es el pedagógico mostrando debilidad en todos los demás factores, principalmente en la desmotivación.

Después de tener esta perspectiva se recomienda la implementación de estrategias pedagógicas y organizacionales para el mejoramiento de las condiciones del clima organizacional en la Universidad, así como la interpelación positiva en el rendimiento académico de los estudiantes. Es necesaria la resignificación de la gestión de los procesos directivos y académicos para responder a las necesidades de todos los actores sociales y generar un ambiente laboral más estable para la institución.

\section{REFERENCIAS BIBLIOGRÁFICAS}

Agudelo, C., Martínez, L., \& Ortiz, I. (2013). Gestión del conocimiento: un activo intangible a través de la investigación. Praxis, 9, 108-113. Recuperado de http://revistas.unimagdalena.edu.co/index.php/praxis/article/ view $/ 750 / 694$

Anzola, O. (2003). Una mirada de la cultura corporativa. Bogotá: Universidad Externado de Colombia.

Azpiazu, L., Esnaola, I., \& Ros, I. (2014). Factores contextuales y variables individuales en el ajuste escolar. International Journal of Developmental and Educational Psychology. Revista INFAD de Psicología, 6(1), 327-336. Recuperado de http://infad. eu/RevistaINFAD/OJS/index.php/IJODAEP/ article/view/751

Balza, V. (2013). La espiral viciosa de la pobreza. Clío América, 7(14), 170-176. Recuperado de http://revistas.unimagdalena.edu.co/index. php/clioamerica/article/view/761

Cárdenas, L., Arciniegas, Y., \& Barrera, M. (2009). Modelo de intervención en clima organizacional. International Journal of Psychological Research, 2(2), 121-127. Recuperado de http://www.redalyc.org/articulo.oa?id $=299023513005$

Chávez, N. (2006). Introducción a la investigación educativa. Cuarta edición. Maracaibo: Gonzales, S.A.T.

Daza, A., Charris, A., \& Viloria, J. (2015). Competencias específicas de los administradores como: factor de desarrollo. Dimensión Empresarial, 13(2), 275-292. Recuperado de https://www.uac.edu.co/images/stories/ publicaciones/revistas_cientificas/dimensionempresarial/volumen-13-no-2/articulo15.pdf

Di Marco, R. (2015). En busca del origen del conocimiento: el dilema de la realidad. Praxis, 11, 150-162. Recuperado de http://revistas.unimagdalena.edu.co/index.php/praxis/article/ view/1561/999 
Edel, R. (2003). El desarrollo de habilidades sociales ideterminan el éxito académico?. Revista electrónica: Red Científica: Ciencia, Tecnología y Pensamiento. En red. Recuperado en: http://www.redcientifica.com/doc/ doc200306230601.html

Fernández, A. (2013). La motivación educativa versus rendimiento. Edición 2. Argentina: LAURES.

García, M. (2009). Clima Organizacional y su Diagnóstico: Una aproximación Conceptual. Revista Cuadernos de Administración, 25(42). Recuperado de http://revistalenguaje.univalle. edu.co/index.php/cuadernosadmin/article/ view/695/2526

Gómez, D. (2014). Apuntes desde la ciencia económica iel sujeto racional o el sujeto complejo? Clío América, 8(15), 83-89. Recuperado de http://revistas.unimagdalena.edu.co/index. php/clioamerica/article/view/831/756

González, M. C., \& Tourón, J. (1992). Autoconcepto y rendimiento escolar: sus implicaciones en la motivación y en la autorregulación del aprendizaje. Pamplona: EUNSA.

Hernández, R.; Fernández, C., \& Baptista, P. (2006). Metodología de la investigación. Cuarta Edición. México: Mc Graw Hill.

Jiménez, M., \& López, E. (2009). Inteligencia emocional y rendimiento escolar: estado actual de la cuestión. Revista Latinoamericana de Psicología, 41 (1), 69-79. Recuperado de http:// www.redalyc.org/articulo.oa?id $=80511492005$

Keogh, B. (2008) Temperamento y rendimiento escolar. ediciones Narcea, Universidad de California.

Litwin, G., \& Stringer, R. (1978). Motivation y Clima Organizacional. Boston: Harvard Business School.

López, L. (2013). Inteligencia Emocional y Rendimiento Académico en Estudiantes de Conservatorio de Música María de Molina (Tesis Doctoral). Universidad Nacional de Educación a Distancia, Chile.

Martínez, A. (2013). Emergencias de cambio: entre el modelo pedagógico tradicional y la necesidad de aprendizajes significativos. Praxis, 9, 73-82. Recuperado de http://revistas.unimagdalena.edu.co/index.php/praxis/article/ view/746/690
Méndez, C. (2001). Metodología: guía para elaborar diseños de investigación en ciencias económicas contables y administrativas. Bogotá: Mc Graw Hill.

Mizola, A. (2005). Factores que Inciden en el rendimiento escolar en Bolivia. Universidad de Chile.

Pérez de Maldonado, I. (2006). El clima y la satisfacción en el trabajo, como fundamentos del éxito en la empresa de principios del próximo milenio. Conferencia presentada en el I Simposio Colombiano sobre Clima Organizacional. Bogotá.

Robbins, S. (2004). Comportamiento Organizacional. México: Prentice Hall.

Saffle, N. (2006) Autoestima y rendimiento escolar. Primera edición. Santiago de Chile: Lone (sol).

Sánchez, J. (2010). Hacia un paradigma emergente de la planeación: Resignificación desde las instituciones universitarias. Santa Marta: Editorial Unimagdalena.

Sánchez, J., Linero, D., \& Martínez, M. (2014). Ocultamiento del discurso pedagógico frente al discurso administrativo en la gestión de las organizaciones educativas. Clío América, 8(15), 36-46. Recuperado de http://revistas. unimagdalena.edu.co/index.php/clioamerica/ article/view/828

Sierra Bravo, R. (2005). Técnicas de investigación social: teoría y ejercicios. Madrid: Paraninfo.

Tamayo \& Tamayo, M. (2006). El proceso de la investigación científica. México: Limusa.

Ureña, Y., Jiménez, I., Mejía, A., \& Vilarete, J. (2014). Cultura innovativa: prospectiva de las condiciones estratégicas para el desarrollo del capital humano. Clío América, 8(16), 195-200. Recuperado de http://revistas.unimagdalena.edu.co/index.php/clioamerica/article/ view/1353

Vega, M., Salazar, C., Huerta, P., \& Nuñez, A. (2008). Clima organizacional y satisfacción laboral en organizaciones del sector estatal (Instituciones públicas) Desarrollo, adaptación y validación de instrumentos. Universum, 23(2), 66-85. Recuperado de http://www. scielo.cl/scielo.php?script = sci_arttext\&pid $=\mathrm{S} 0718-23762008000200004$ 
Viloria, J., Bertel, M., \& Daza, A. (2015). Percepciones estudiantiles sobre el proceso de acreditación por alta calidad del Programa de Administración de Empresas de la Universidad del Magdalena. Praxis. 11, 89-102. Recuperado de http://revistas.unimagdalena.edu.co/ index.php/praxis/article/viewFile/1557/995
Viloria, J., Pertúz, S., Daza, A., \& Pedraza, L. (2016). Aproximación al clima organizacional de una empresa pro- motora de salud. Revista Cubana de Salud Pública, 42(1). Recuperado de http://www.revsaludpublica.sld.cu/index. php/spu/article/view/592/481 